\title{
AIRPLANE ANTENNA CONSTANTS ${ }^{1}$
}

\author{
By J. M. Cork
}

\section{CONTENTS}

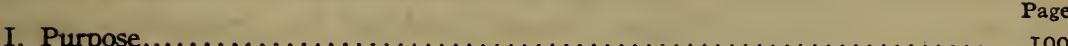

II. Capacity and resistance measurements. $\ldots \ldots \ldots \ldots \ldots \ldots \ldots \ldots \ldots \ldots$ I9

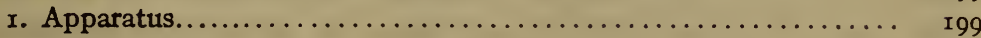

2. Manipulation..................... 200

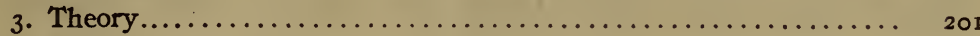

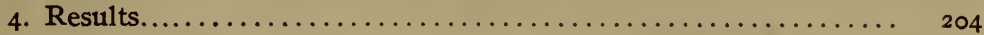

(a) Fixed antennas.............................. 204

(b) Trailing antennas..........................

III. Directional effect.................................... 2 II

\section{PURPOSE}

The purpose of this work was to obtain general information regarding the effective capacity, effective resistance, true capacity, true inductance, and wave length, as well as the transmitting directional effect of various types of airplane antennas. In addition to trailing antennas of one, two, and four wires, the constants of various fixed antennas were measured in an attempt to find a satisfactory. substitute for the trailing wire at short wave lengths.

\section{CAPACITY AND RESISTANCE MEASUREMENTS}

\section{APPARATUS}

All measurements were made in flight, using a continuous wave oscillator feeding directly into the antenna. A measurement of the effective resistance and effective capacity of each antenna was made at several different wave lengths, from which resistance wave-length and capacity wave-length curves were drawn. A wiring diagram of the test set used is shown in Fig. I. It may be observed that this is the familiar Colpitts circuit, in which the antenna takes the place of the regular coupling condenser. By changing $K_{1}$ from $A$ to $B$ a dummy antenna is substituted for the

1 The data given in thjs paper were obtained by the writer while an officer in the Signal Corps, United States Army, and js publjshed with the consent of this organization. 
one under test. This consisted of a calibrated straight wire resistance variable in steps of $0.1 \mathrm{ohm}$, and a variable condenser calibrated for both capacity and resistance at various wave lengths and angular settings. The oscillator and dummy antenna, together with a wave meter of suitable range, were mounted on the bakelite panel of a set box which fitted in the front of the cockpit, convenient for reading condensers and ammeters. By switch $K_{2}$ the wave meter could be open circuited and thus absorb no energy. All condensers were shielded by grounded copper cases. For measurements at short wave lengths the inductance was made by winding No. I4 wire on a wooden toroidal core, having taps every three turns for various wave lengths. This

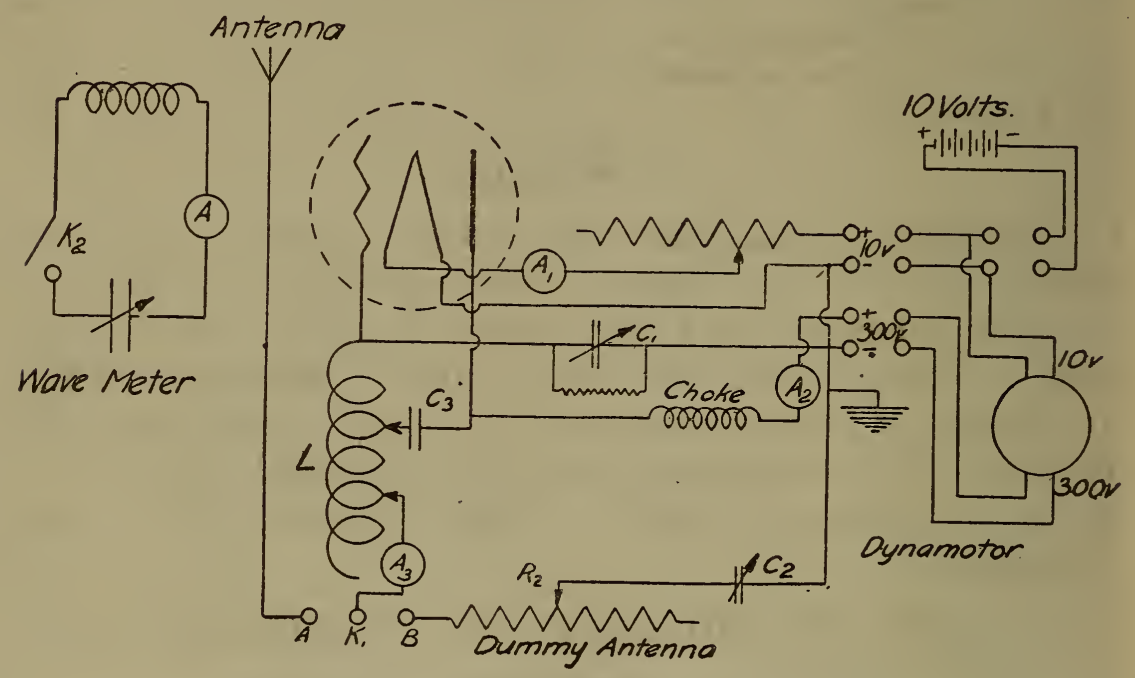

FIG. I.-Wiring diagram of airplane antenna test set

eliminated to a large degree all stray magnetic fields. Power was supplied by a ro-volt storage battery and a 300 -volt dynamotor, battery driven. $A_{1}, A_{2}, A_{3}$ are ammeters in filament, dynamotor, and oscillating circuits, respectively. A grid leak of 50 oooohms shunted the variable grid condenser. All leads carrying oscillations were made as short as possible to avoid stray capacities.

\section{MANIPULATION}

The effective capacity was determined by adjusting the inductance taps and grid condenser for good oscillation at approximately the desired wave length, with the antenna in circuit, then setting the wave meter at resonance. The switch $K_{1}$ is next turned to 
$B$ and the dummy condenser adjusted until the wave meter is again in resonance. From the condenser calibration the capacity at this wave length may be obtained. To find the effective resistance, having set capacities as above, the wave meter is open circuited by opening $K_{2}$ and the reading of $A_{2}$ noted with $K_{1}$ at $A$; now turning $K_{1}$ to $B, R_{2}$ is adjusted until the $A_{2}$ reading is duplicated. From the calibration of $R_{2}$ the effective resistance is obtained. This furnishes a very accurate and sensitive means for the determination of effective antenna resistance. By varying the grid condenser the range of critical sensitivity may be set at any

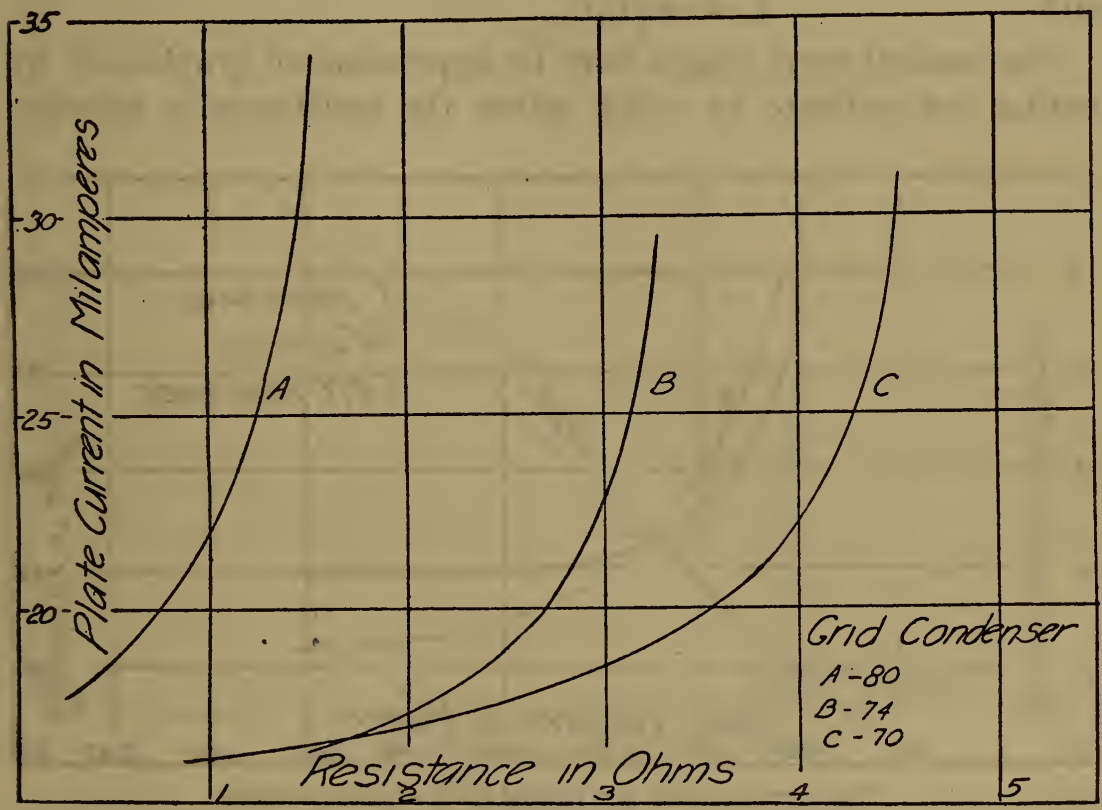

Fig. 2.-Sensitivity of test set for changes in the resistance of the oscillating circuit

resistance value desired. Fig. 2 shows the effect of changing the grid condenser setting, giving at the critical range a change of more than I milliampere for $0 . \mathrm{I} \mathrm{ohm}$ change in resistance. Thus if the resistance be first found approximately, the grid condenser may be set so as to make the point fall at a sensitive position.

\section{THEORY}

Fig. 3 shows typical wave-length capacity and wave-length resistance curves. At longer wave lengths the effective capacity becomes asymptotic to a value which is the natural or true capacity $C_{0}$. Therefore, since at the natural wave length the 
total reactance of the antenna is zero (i. e., for an infinitesimal applied voltage a large current flows in the antenna), $\frac{\mathrm{I}}{\omega C}$ effective is zero. But the effective capacitance at all times equals the difference between the natural capacitance and natural inductive reactance, or

$\begin{array}{ll} & \frac{\mathrm{I}}{\omega C} \text { effective }=\frac{\mathrm{I}}{\omega C_{\mathrm{o}}^{\circ}}-L_{\mathrm{o}} \omega \\ \text { or, } & L_{\mathrm{o}}=\frac{\mathrm{I}}{\omega^{2}}\left(\frac{\mathrm{I}}{C_{\mathrm{o}}}-\frac{\mathrm{I}}{\mathrm{C}} \text { effective }\right) \\ \text { and } & \lambda_{\mathrm{o}}=2 \pi \sqrt{L_{0} C_{\mathrm{o}}}\end{array}$

The natural wave length may be approximated graphically by noting the ordinate to which either the resistance or effective

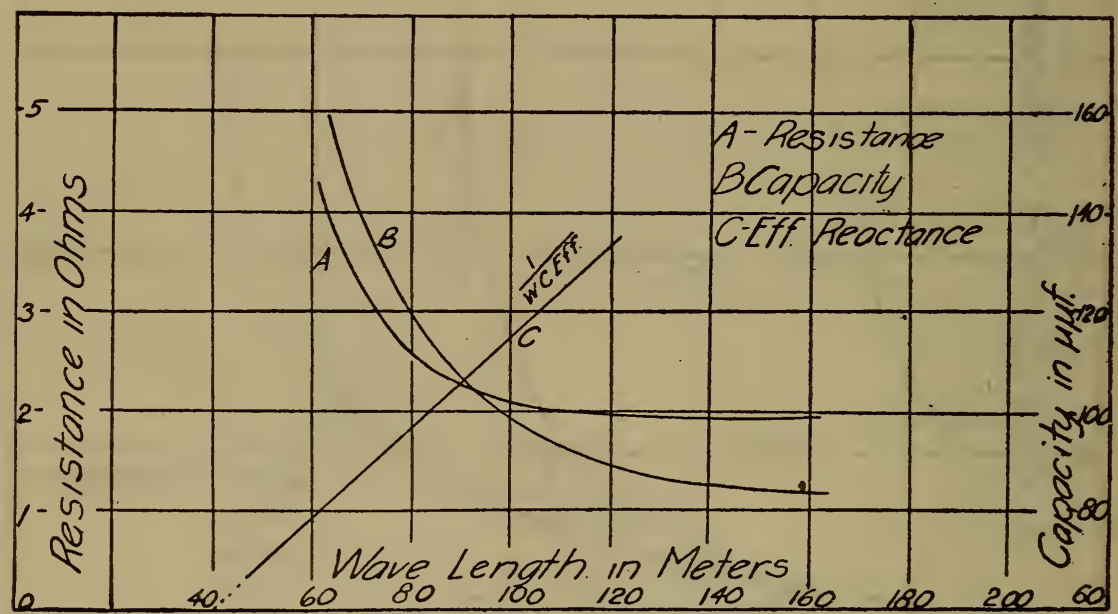

FIG. 3. - Constants of a typical fixed airplane antenna

capacity curves are asymptotic; or, better still, since at wave lengths above the fundamental the capacity reactance predominates, by plotting an effective capacitance wave-length curve as shown in $C$, Fig. 3, practically a straight line is obtained. This may be extended to intersect the wave-length axis, thus giving the natural wave length. Instead of the effective capacitance. $\frac{\mathrm{I}}{\omega C}$ effective, $\frac{\lambda}{C}$ effective may be used as well, since not actual reactance values but only the intersection of the line with the axis is desired.

The curve shown in $B$, Fig. 3 , gives effective antenna resistance. This, of course, is the resultant of several components. Fig. 4 
shows the manner in which the components might be expected theoretically to change with increasing wave length; $b$ represents the pure ohmic resistance due to the metallic conductors. At long wave lengths this is practically a constant, but increases slightly at short wave lengths due to skin effect, shown by $b^{1}$. If $R^{1}$ denotes the sum of the direct-current resistance $R$, plus the

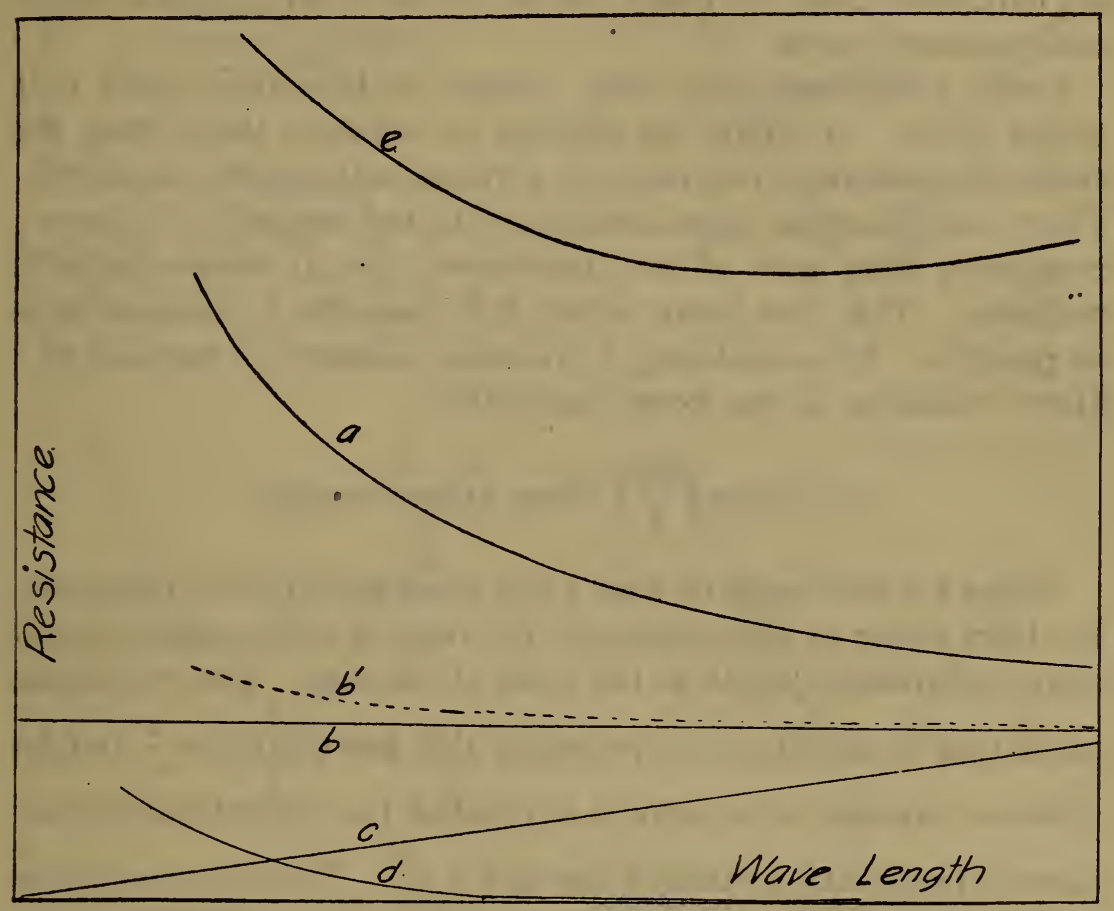

FIG. 4.-Components of antenna resistance

additional resistance due to skin effect, at very short wave lengths an approximate formula for the variation of $R^{1}$ is

$$
R^{1}=R \frac{K}{\lambda^{1 / 2}}
$$

But as $\lambda$ increases, the approximate formula ${ }^{2}$ becomes

$$
R^{1}=R\left(\mathrm{I}+\frac{K}{\lambda^{2}}-\frac{K^{2}}{\lambda^{4}}+\ldots\right)
$$

Curve $c$ represents resistance due to dielectric loss. In this respect an antenna like a condenser has a phase angle, and may 
bc considered as a resistance and a pure capacity in series, the resistance increasing as the first power of the wave length. The location of the dielectric producing the loss has been the subject of considerable investigation. Dr. J. M. Miller ${ }^{3}$ has shown that the earth, as was commonly assumed, is rarely the cause, but rather dielectrics in the proximity of the antenna. It is of interest to observe that this effect was not noted in any of the airplane measurements made.

Curve $d$ represents any eddy current or hysteresis losses that might occur. It would be difficult to separate these from the radiation resistance, but they are perhaps both entirely negligible. They would increase approximately with the frequency. Curve $a$ represents that part of the resistance due to electromagnetic radiation. It is this factor which it is desirable to have as large as possible. By considering a grounded antenna as one-half of a Hertz oscillator, it has been shown that

$$
R_{\mathrm{a}}=\mathrm{I} 60 \pi^{2}\left(\frac{a h}{\lambda}\right)^{2} \text { ohms approximately }
$$

Where $h$ is the length of wire, $\lambda$ the wave length and $a$ represents the form factor or approximately the ratio of the average current along the antenna length to the value at the base. For an antenna oscillating at its natural wave length this has the value $\frac{2}{\pi}$, but for a loaded antenna as is common in practice the current distribution curve is practically a straight line and $a=\frac{\mathrm{I}}{2}$. Then for an airplane antenna of length, say, 200 feet, transmitting at a wave length of 400 meters, $R_{\mathrm{a}}=6 \mathrm{ohms}$ approximately, and at 1000 meters $R_{\mathrm{a}}=\mathrm{I}$ ohm approximately.

\section{RESULTS}

(a) Fixed Antennas.-Measurements were made upon fixed antennas over a range in wave length from 60 to 160 meters. In all cases the airplane wires bonded together were used as ground.

Fig. 5 shows some of the arrangements of wires measured. Most of these were first measured close to the plane, then raised on 2 -foot and then 4 -foot masts. The change from close to plane to 2 -foot mast produced a much larger decrease in natural capacity than the change from 2 to 4 foot masts. The raise in height, 
however, is accompanied by an increase in radiation resistance. Typical wave-length capacity and wave-length resistance curves of the fixed antennas are shown in Fig. 3. This is for an antenna
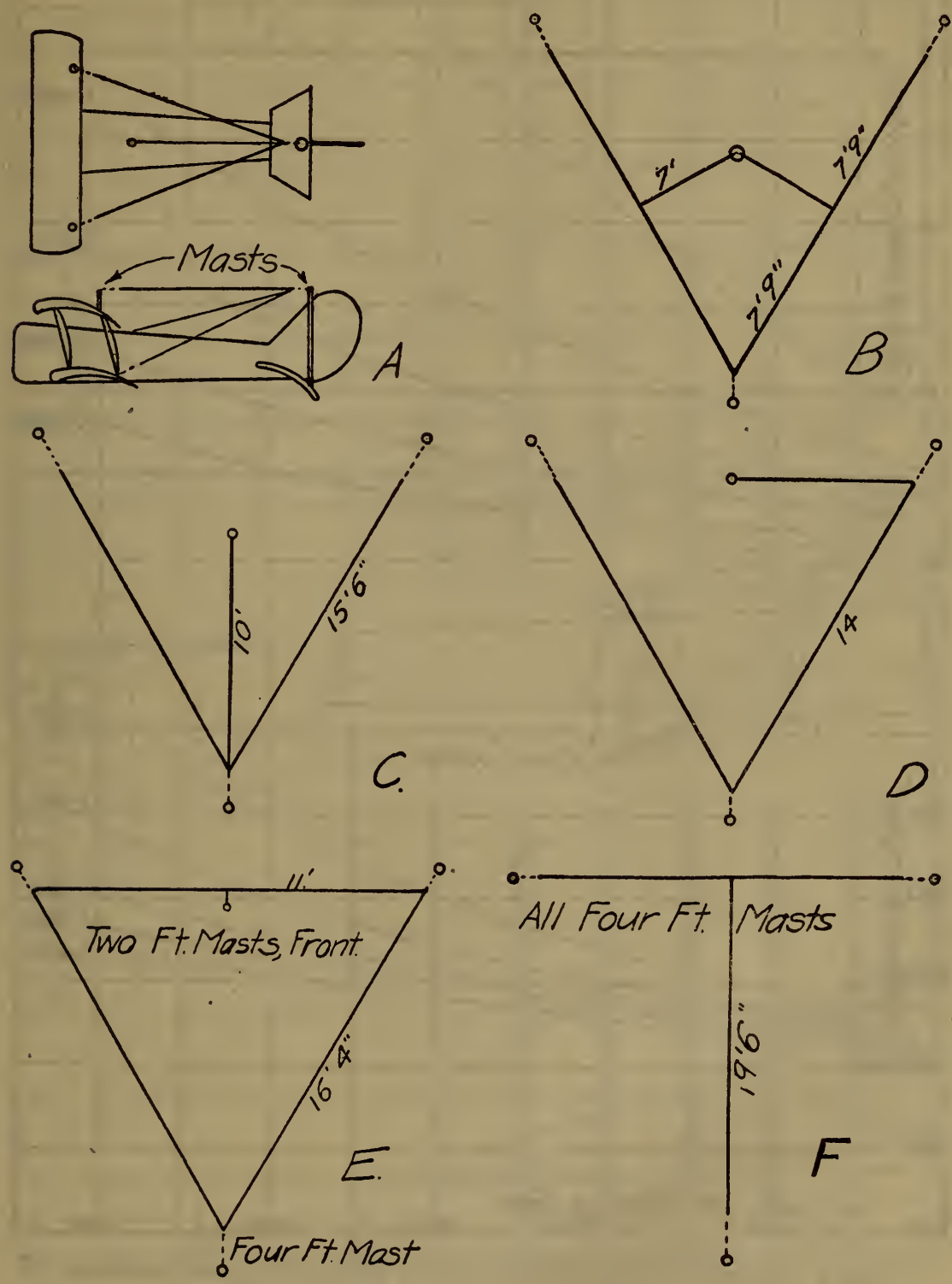

FIG. 5.-Types of fixed airplane antennas

of the form $D$ shown in Fig. 5 raised on masts 2 feet high. The height of mast used is determined by the maximum wind resistance aliowable, as well as by the electrical characteristics. Antenna 
form $D$, Fig. 3 , is about equivalent in characteristics to a single 45 -foot trailing wire except that it is not so directional in trans-

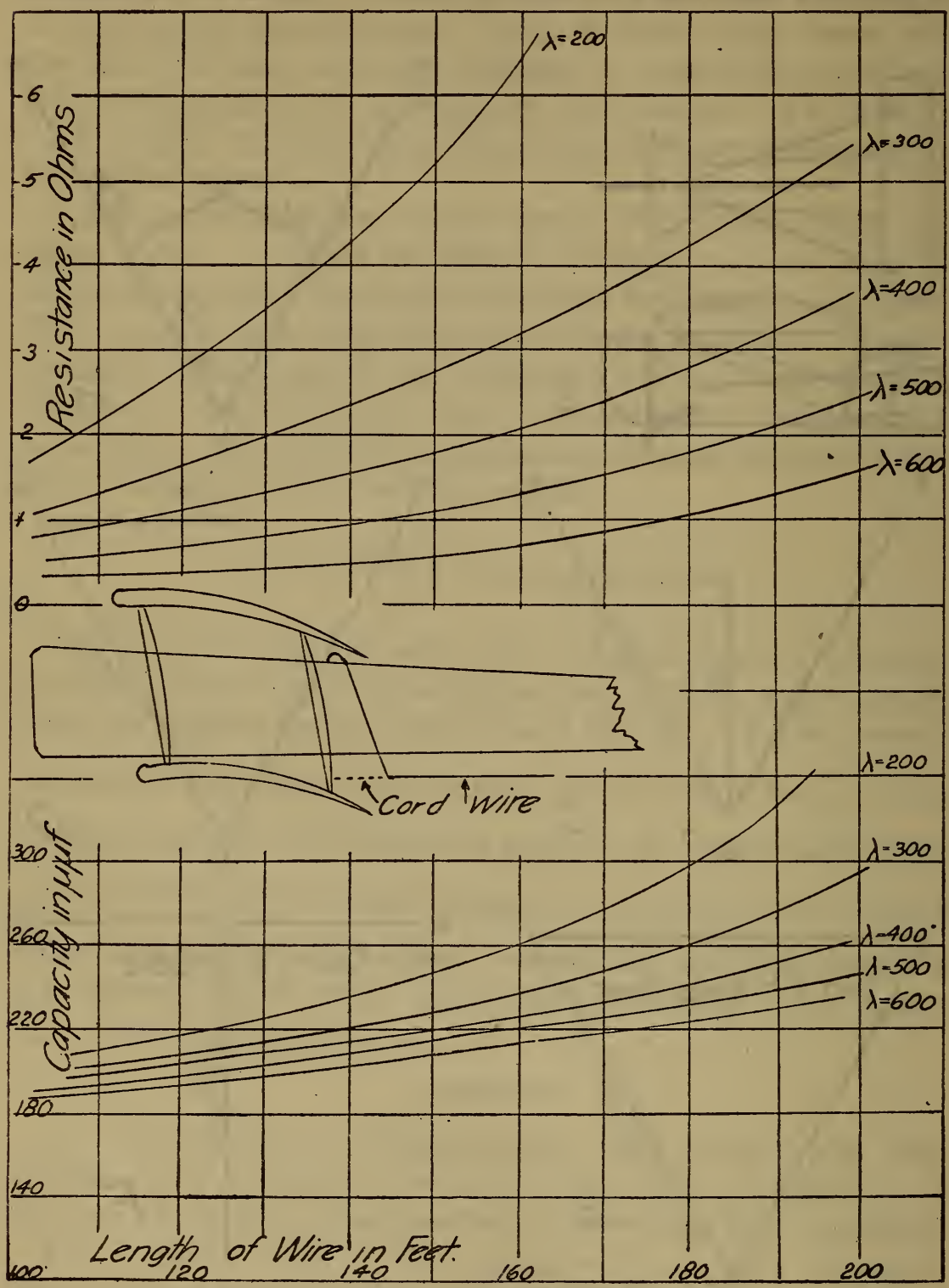

FIG. 6.-Summary of the constants for various lengths of single-wire antennas

mitting. Using this antenna and a single tube oscillator, voice signals have been heard at a distance of 5 miles from plane to ground, using a three-tube receiver. 
(b) Trailing Antennas.-Measurements were made upon trailing wires over a wave-length range from 100 to 600 meters. The single-wire antenna was attached by an insulator and 40 -inch

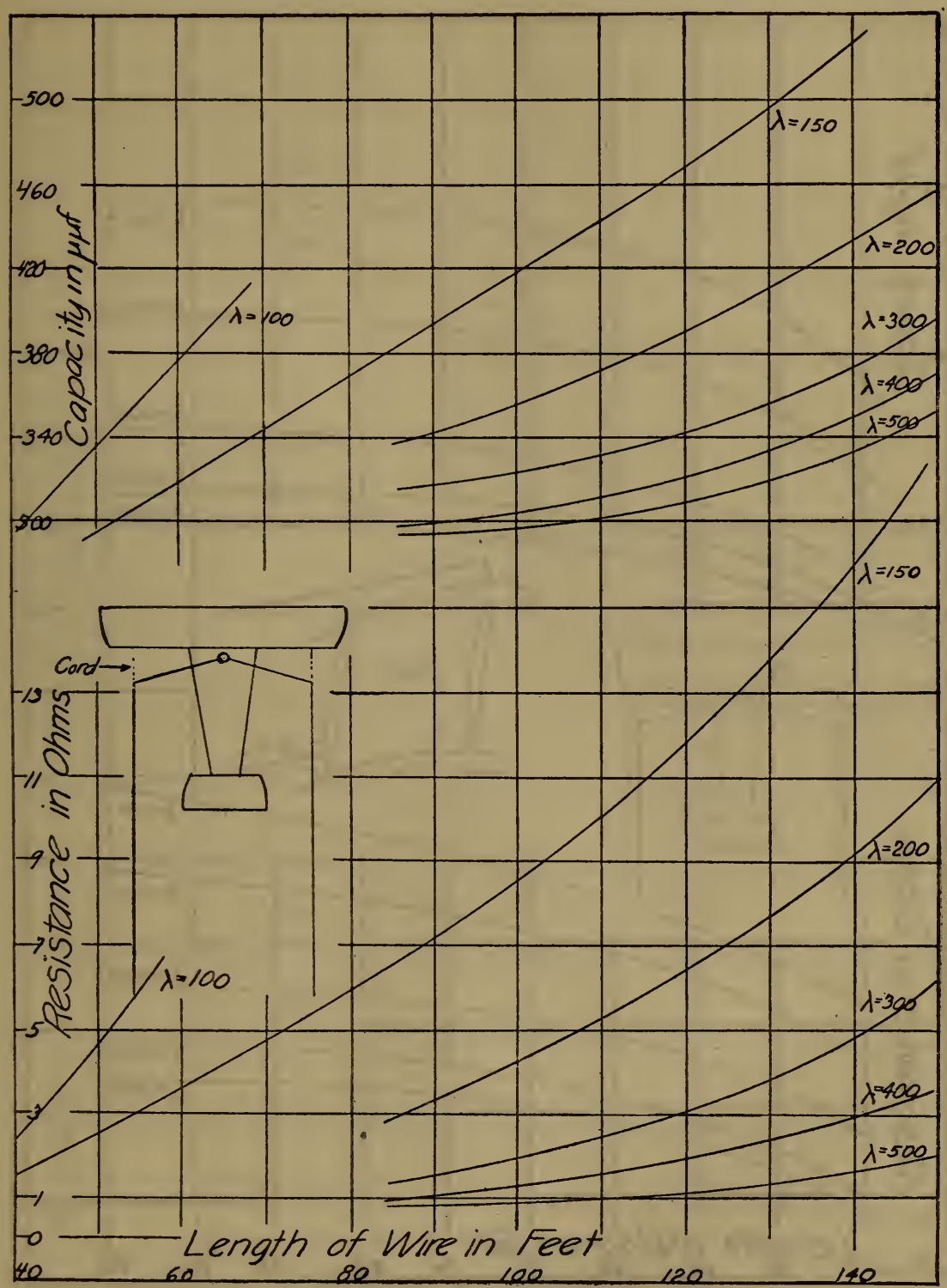

Fig. 7.-Summary of the constants for various lengths of two-wire antennas

string to the inside strut of the plane. The position of the lead in wire is quite an important factor in determining the total capacity of the antenna and should be kept as far as possible from 
the wire network of the machine. Various lengths of wire were thus used, and in Fig. 6 the results are summarized by plotting capacity length of wire and resistance length of wire curves at

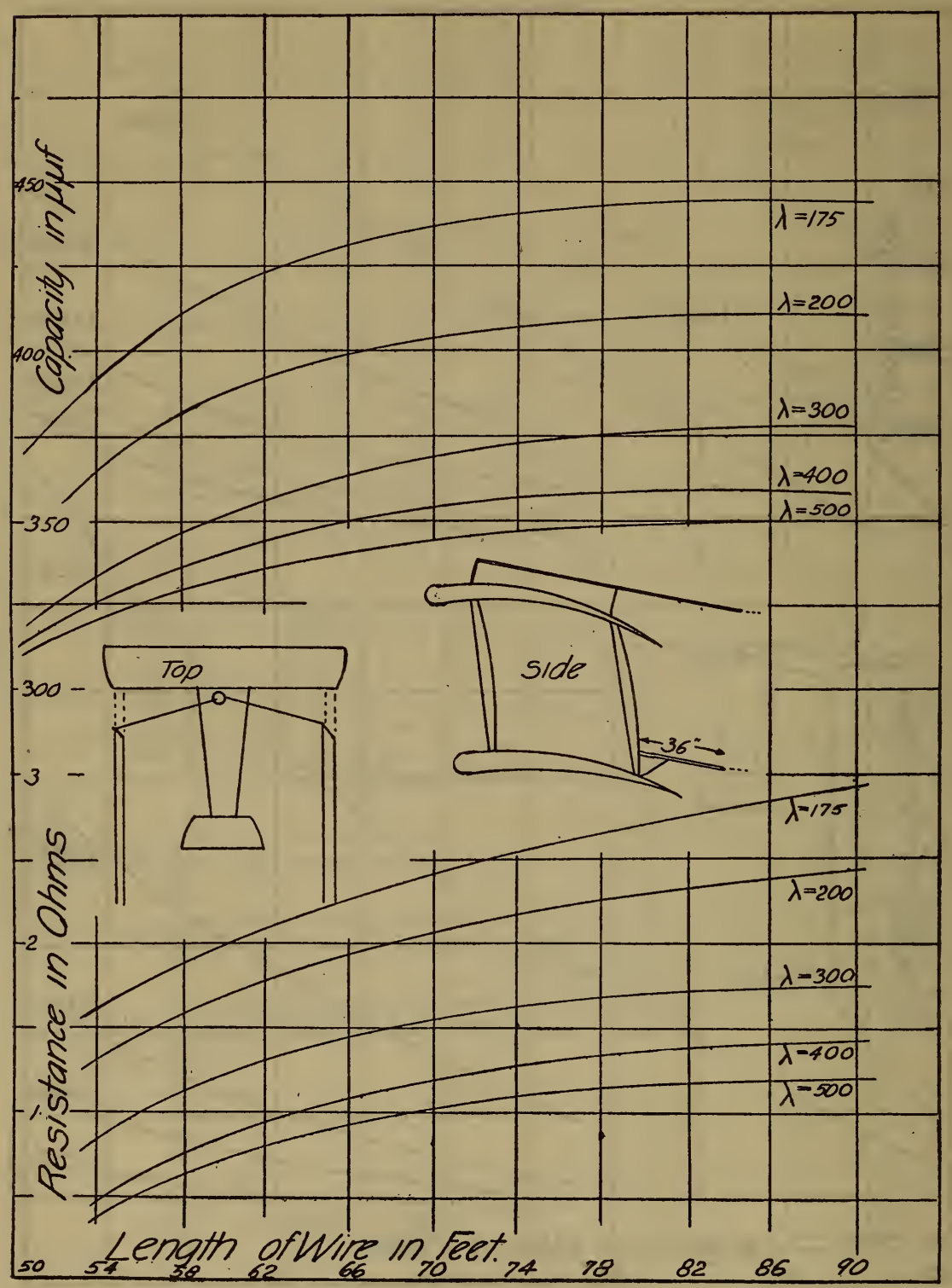

FIG. 8.-Summary of the constants for various lengths of four-wire antennas

various wave lengths. Thus the characteristics for any wire length may be obtained by taking the ordinates of the various wave-length curves at that abscissa. Fig. 7 shows a like sum- 
mary for a two-wire antenna attached as shown in the figure, using the wires in parallel against airplane wires as ground. Data were also obtained using one wire as the ground and the

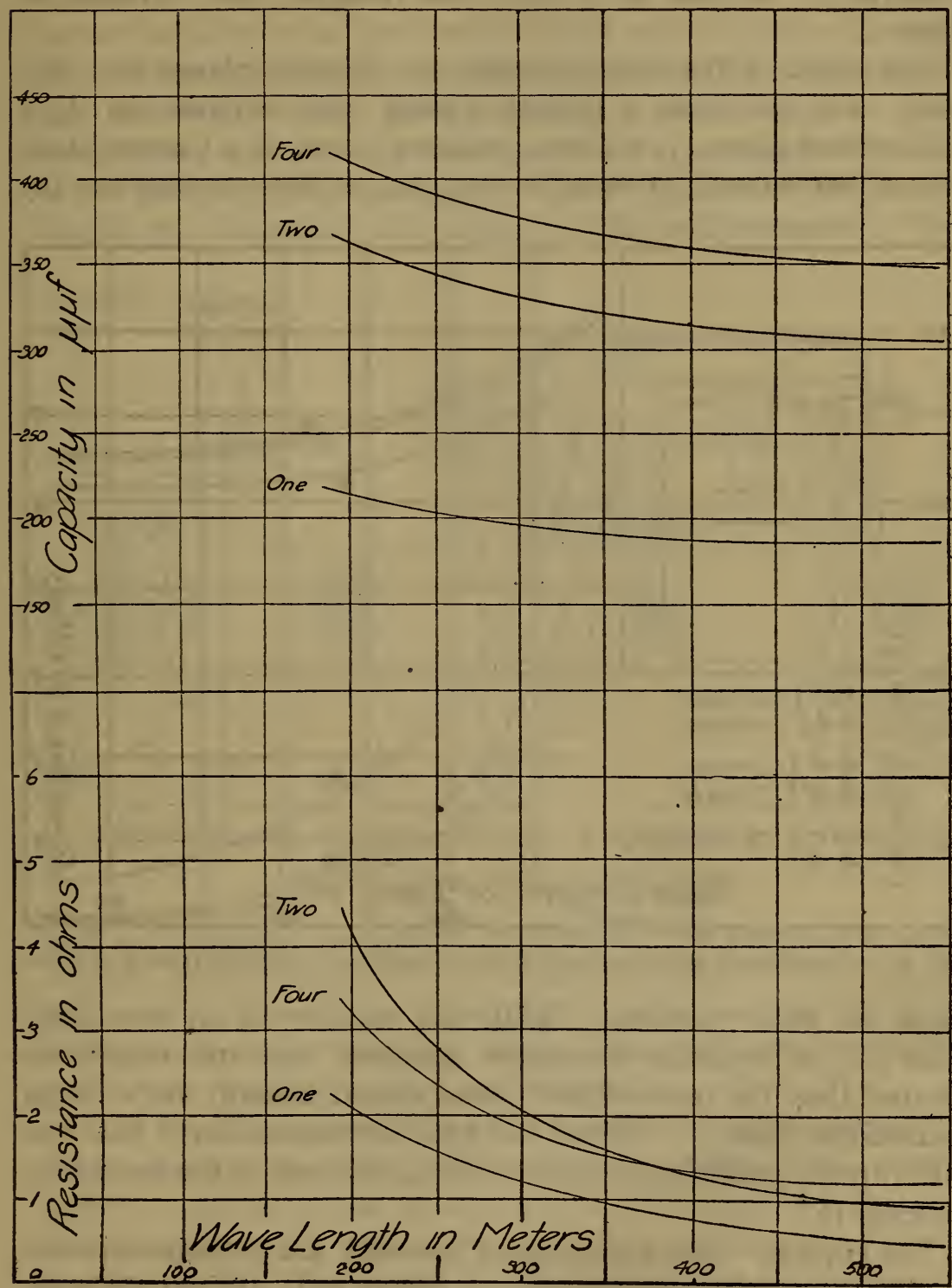

Fig. 9-Comparison of the constants of a roo-foot trailing antenna of one, two, and four wires

other as antenna. The capacity in this case is very greatly reduced, and the radiation resistance is also less. Fig. 8 shows a summary of a four-wire antenna, using machine as ground. This 
antenna has a very large capacity, as would be expected. Fig 9 shows a comparison of the characteristics of a Ioo-foot single, two, and four wire antenna. The resistance could in no case be observed to increase at longer wave lengths due to dielectric losses.

The effect of the same antenra on different planes was also tried. Fig. Io shows a typical trailing wire antenna on $A_{3}$ a DeHaviland plane, $A_{2}$ a Curtiss training plane, $A_{1}$ a Curtiss plane with a fine network of wires in wings placed there to improve the

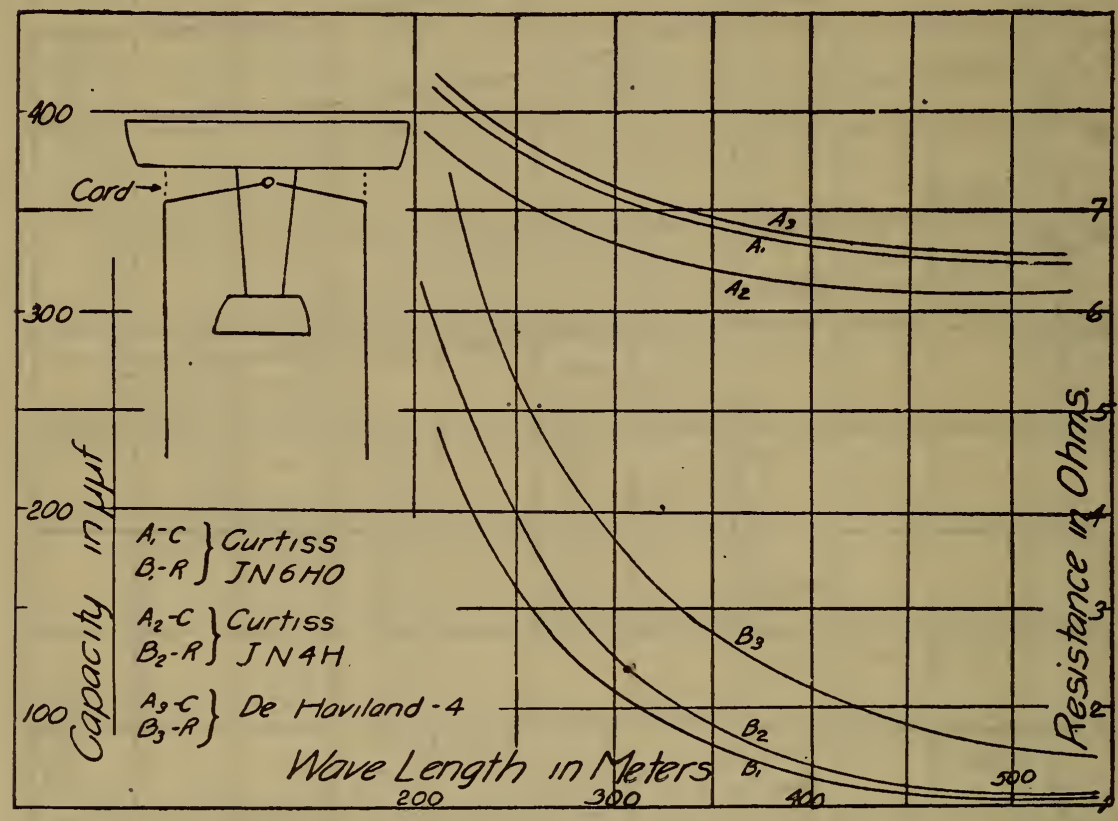

FIG. Io.-A comparison of the constants of the same antenna on different types of airplanes

plane for radio purposes. While the capacity of $A_{6}$ was larger than $A_{2}$, its radiation resistance appeared less and results indicated that the unmetallized plane should actually be a better transmitter than $A_{1}$. While the final demonstration of this was interrupted, preliminary results with actual sets in use seemed to indicate it.

The capacity values mentioned herewith are perhaps accurate to within Io micro-microfarads, as repeat tests did not differ by this amount. The resistance values, however, may be in error by 20 per cent, especially at the longer wave-length values. 


\section{DIRECTIONAL EFFECT}

In order to determine the directional transmitting effect of the various antennas, a receiving set, as shown in Fig. I I, was connected to a symmetrical vertical wire antenna. The receiving set consisted of a detector tube with grid at positive potential and a three-stage audio-amplifier with tubes having a negative grid potential. In the plate circuit of the last tube was placed the primary circuit of a transformer with secondary connected to the heater of a thermocouple. The output emf of the thermocouple was connected to a sensitive Paul microammeter. Then

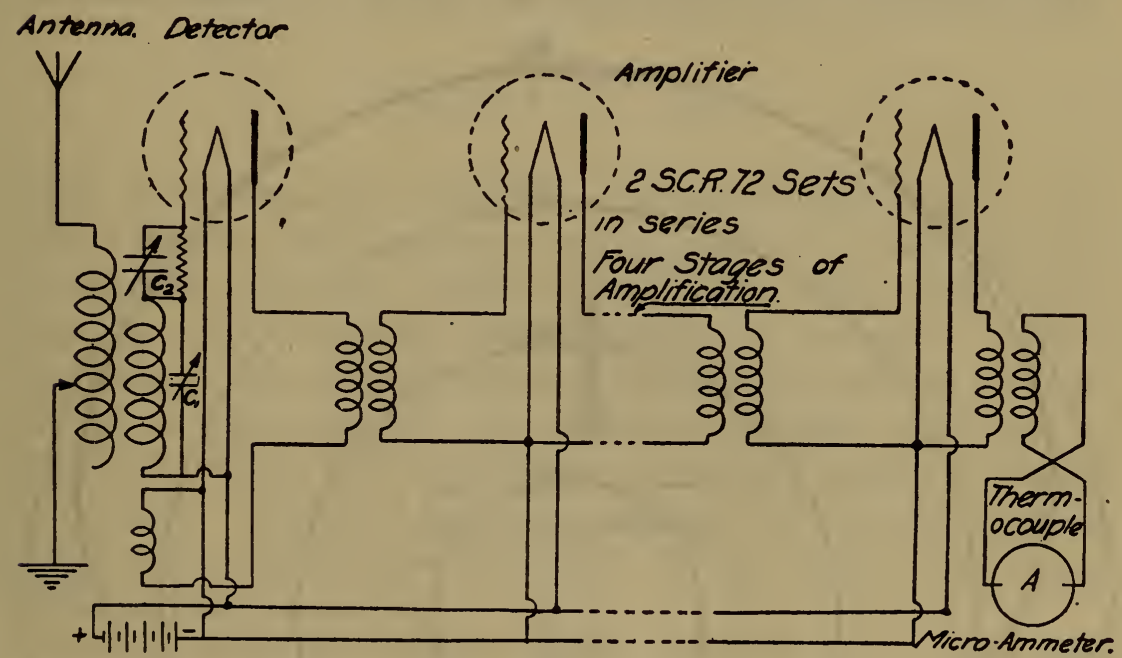

FIG. II.-Wiring diagram of receiving set

with a modulated undamped transmitter the plane flew over a marked distant point in the different directions of the compass. When directly over the point the operator shut off the transmitter, and at the receiving station the microammeter reading just before dropping to zero was recorded.

As it would be very difficult to compute mathematically the exact relation between microammeter reading and received energy, since it would necessitate knowing just where on its characteristic curve each tube was being worked, the following test was made. The current in the transmitting antenna was set at various known values, as $I_{1}$ and $I_{2}$, and the corresponding micro-ammeter readings, $C_{1}$ and $C_{2}$ taken, wave length and direction remaining the same; the constants should remain almost the 
same if the amplitude of the incoming wave did not vary greatly. Thus, assume-

and,

$$
I_{1}=K C_{1}^{\alpha}
$$

then

$$
I_{2}=K C_{2}^{\alpha}
$$

$$
a=\frac{\log I_{1}-\log I_{2}}{\log C_{1}-\log C_{2}}
$$

This means then that if the microammeter readings are to be proportional to transmitted currents, they must be raised to the a power, or, if proportional to energy to the 2 a power.

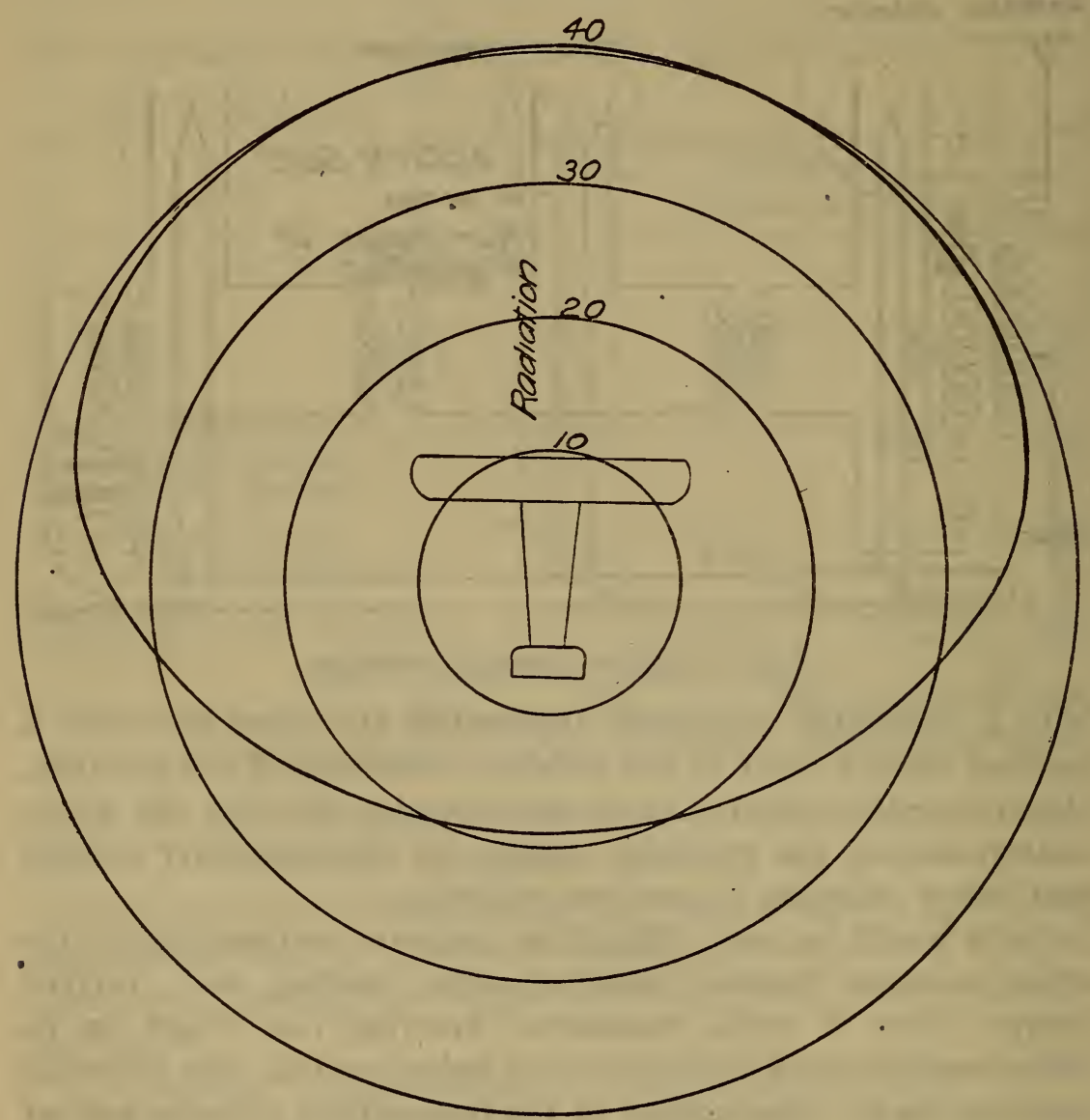

FIG. 12.-Directional effect of a two trailing wire airplane antenna

Making this correction in the data, the trailing wire antenna is found to be quite directional, giving about twice the radiation in the direction of motion that it does in the reverse direction, as 
shown by Fig. I2. The fixed antennas are not decidedly directional.

The directional effect is probably a function of the relation between the antenna length and the wave length. A theoretical explanation of this effect is quite difficult. The electrostatic component of the electromagnetic field at a distance $r$ from a radiator having a length component perpendicular to $r$ of $h \sin a$ and a current of $I_{0}$ is ${ }^{4}$

$$
E_{1}=2 \pi V_{2} \frac{h \sin \alpha}{\lambda} \frac{I_{0}}{r} \text { where } V_{2}=3 \times 10^{10}
$$

and the magnetic component at right angles and in the same phase

$$
M_{1}=2 \pi \frac{h \sin \alpha}{\lambda} \frac{I_{0}}{r}
$$

From these equations currents parallel with the antenna wire should have no effect upon the energy received at points directly in line with the wire, forward or backward. This energy must, then, be due to radiation from. perpendicular currents. It may be, however, that the inclination of the antenna with the horizontal line of flight presents a perpendicular radiating component to a point forward, which would be less or entirely lacking to a point in the rear. In general, any explanation of the directive effect of the Marconi bent antenna when used over sea water would apply to the airplane antenna.

This opportunity is taken to express thanks to A. A. Oswald, of the Western Electric Co., for valuable suggestions given, and to those officers and enlisted men of the Signal Corps by whose authority and assistance this work was made possible.

WASHINGTON, February 2 I, I9I 9.

"J. Zennecik, Wireless Telegraphy, p. 35; Feb. 21, rgrg. 